\title{
Force-mediated proinvasive matrix remodeling driven by tumor-associated mesenchymal stem-like cells in glioblastoma
}

\author{
Eun-Jung Lim ${ }^{1, \#}$, Yongjoon Suh ${ }^{1, \#}$, Seungmo Kim ${ }^{1}$, Seok-Gu Kang ${ }^{2}$ E Su-Jae Lee ${ }^{1, *}$ \\ ${ }^{1}$ Department of Life Science, Research Institute for Natural Sciences, Hanyang University, Seoul 04763, ${ }^{2}$ Department of Neurosurgery, \\ Brain Tumor Center, Severance Hospital, Yonsei University College of Medicine, Seoul 03722, Korea
}

In carcinoma, cancer-associated fibroblasts participate in force-mediated extracellular matrix (ECM) remodeling, consequently leading to invasion of cancer cells. Likewise, the ECM remodeling actively occurs in glioblastoma (GBM) and the consequent microenvironmental stiffness is strongly linked to migration behavior of GBM cells. However, in GBM the stromal cells responsible for force-mediated ECM remodeling remain unidentified. We show that tumor-associated mesenchymal stem-like cells (MSLCs) provide a proinvasive matrix condition in GBM by force-mediated ECM remodeling. Importantly, CCL2-mediated Janus kinase 1 (JAK1) activation increased phosphorylation of myosin light chain 2 in tMSLCs and led to collagen assembly and actomyosin contractility. Collectively, our findings implicate tMSLCs as stromal cells providing force-mediated proinvasive ECM remodeling in the GBM microenvironment, and reminiscent of fibroblasts in carcinoma. [BMB Reports 2018; 51(4): 182-187]

\section{INTRODUCTION}

Glioblastoma (GBM) is the most common and aggressive primary brain tumor in adults (1). Despite the advent of modern medical treatments, median survival is still less than 15 months, and long-term survival is extremely rare (2). The poor prognosis of GBM is attributed to the diffuse and unrelenting infiltration of tumor cells throughout the brain despite optimal treatment $(2,3)$.

Although there are many mechanisms involved in invasion of GBM cells, extracellular matrix (ECM) remodeling has

*Corresponding author. Tel: +82-2-2220-2557; Fax: +82-2-22990762; E-mail: sj0420@hanyang.ac.kr

${ }^{\text {\#} T h e s e ~ a u t h o r s ~ c o n t r i b u t e d ~ e q u a l l y ~ t o ~ t h i s ~ w o r k . ~}$

https://doi.org/10.5483/BMBRep.2018.51.4.185

Received 15 September 2017, Revised 20 October 2017, Accepted 4 December 2017

Keywords: Actomyosin contractility, Glioblastoma, Proinvasive extracellular matrix remodeling, Tumor-associated mesenchymal stem-like cells received much attention due to its direct interaction with GBM cells in the tumor microenvironment. Indeed, infiltration of GBM correlated with the ability to alter the properties of the surrounding extracellular space and to interact with its components $(4,5)$.

Since the role of ECM in cancer progression has begun to be understood $(6,7)$, its importance to GBM has also drawn great attention. Generally, ECM has been regarded as a hurdle that cancer cells must overcome to enter the blood vessels for metastasis to other organs. Accordingly, invasion of metastatic cancer cells throughout ECM has been thought to be enabled by protease-mediated matrix degradation that makes a movement track for migration of cancer cells. In line with this notion, there was a strong correlation between activation of matrix metalloproteinases (MMPs) that degrade ECM and invasiveness of GBM (8). Alternatively, instead of proteasemediated matrix remodeling, cancer cell invasion in ECM environment could be enabled by force-mediated rearrangement of ECM that gives a mechanical stiffness in tumor microenvironment (9-11). Indeed, many tumors, including GBM, are mechanically stiffer than the surrounding stroma $(12,13)$. Interestingly, glioma migration and proliferation were affected by rigidity of ECM (14). Although both protease- and force-mediated ECM remodeling contribute to invasion of cancer cells, the mechanism underlying force-mediated matrix remodeling is largely unexplored in brain tumors.

In the case of carcinoma, ECM is highly produced and remodeled by carcinoma-associated fibroblasts (CAFs) (15). Although soluble factors secreted by CAFs play a role in malignant phenotypes of cancer, CAFs also contributed to invasiveness of cancer cells by force-mediated ECM remodeling using actomyosin contractility through Rho-Rho-kinase (ROCK) signaling (11). Likewise, GBM is also known to be mechanically stiffer than the surrounding parenchyma and force-mediated ECM remodeling has been observed as an important parameter in GBM progression $(6,7)$. In contrast to carcinoma, however, the equivalent stromal cells responsible for the stiffness in GBM remain unknown.

Among stromal cells, mesenchymal stem cells (MSCs) have been recently identified as stromal components in many cancers, including GBM (16-19). Since MSCs have wound tropism and the potential to repair damaged tissues by ECM 
remodeling $(20,21)$, we speculated that tumor-associated mesenchymal stem-like cells (tMSLCs) in GBM may provide mechanical stiffness and facilitate the motility of GBM cells. Accordingly, we investigate whether tMSLCs could generate force-mediated ECM remodeling and provide a proinvasive matrix environment in GBM that is reminiscent of CAFs.

\section{RESULTS}

\section{tMSLCs-induced ECM remodeling enhances invasion of GBM cells}

In a previous study, we established human tMSLCs from glioma specimens (22). Those cells were non-tumorigenic and similar to mesenchymal stem cells (23), based on their spindle-shaped morphology and expression of MSC surface antigens (CD105, CD73, and CD90), but not leukocyte (CD45), endothelial cell (CD31), or pericyte (NG2) markers. In parallel, these cells were able to differentiate into tri-lineage mesenchymal cells, including osteogenic, adipogenic, and chondrogenic cells. To investigate the effect of tMSLCs on invasiveness of GBM cells, we analyzed infiltration of X01 GBM cells in coculture with or without TMSLC0903 in a collagen-based matrix, a similar composition to that of in vivo basement membrane, in which collagen type I and matrigel were mixed and solidified in a growth medium (Fig. 1A).
A

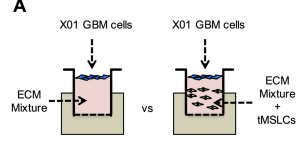

C

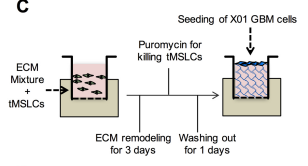

D

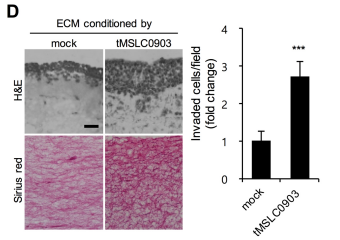

B
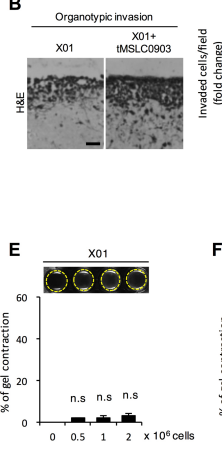
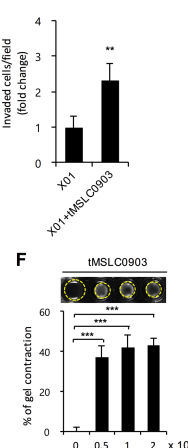

Fig. 1. tMSLCs-mediated ECM remodeling enhances invasiveness of GBM cells. (A, B) Schematic illustration (A) and quantification (B) of GBM cell invasion in collagen-based gel matrix premixed with tMSLC0903. (C) Schematic illustrating the experimental scheme for analysis of GBM cell invasiveness in collagen-based ECM preconditioned by tMSLC0903. (D) H\&E staining (upper panel) and collagen staining with Sirius red (lower panel). Quantification of X01 GBM cells infiltrated into the collagen-based matrix preconditioned by tMSLCs (right panel). Scale bar, $100 \mu \mathrm{m}$. (E, F) Quantification of matrix contraction by X01 GBM cells (E) and tMSLC0903 (F). Upper panel shows scanned images of the contracted gel. Data are presented as mean \pm SD from one of three independent experiments performed. ${ }^{* * P}<0.01$ vs. control; $* * * P<0.001$ vs. control.

Compared to GBM cells alone, invasion of GBM cells was increased by the presence of tMSLCs in the matrix (Fig. 1B). Although tMSLCs stimulate GBM cells with soluble factors or cell-to-cell contact, we were more interested in whether tMSLCs contribute to invasion of GBM cells by their ECM remodeling capability. To clarify, tMSLCs were cultured in a collagen-based matrix and allowed ECM remodeling for 3 days before killing the cells with puromycin, followed by washing out cell debris and soluble factors, and seeding of X01 GBM cells on top of the matrix (Fig. 1C). Importantly, GBM cells were more invasive in the matrix that has been remodeled by tMSLCs than in the matrix where ECM was not remodeled by tMSLCs (Fig. 1D). By collagen staining with Sirius red, we also noticed that collagen staining is intensive in the matrix conditioned by tMSLCs (Fig. 1D). Although the increase in Sirius red staining could be due to the collagen-rich condition, it could be also caused by formation of collagen bundles. Accordingly, we further measured the ability of the tMSLCs to contract collagen matrix. As expected, gel contraction increased as the number of tMSLCs increased, whereas X01 GBM cells demonstrated no capability to contract gel (Fig. 1E, F). Taken together, these results implicate that TMSLCs contribute to invasiveness of GBM cells via force-mediated ECM remodeling.

\section{JAK1 activation is required for ECM remodeling ability of tMSLCs}

Given that tMSLCs promote invasion of GBM cells through

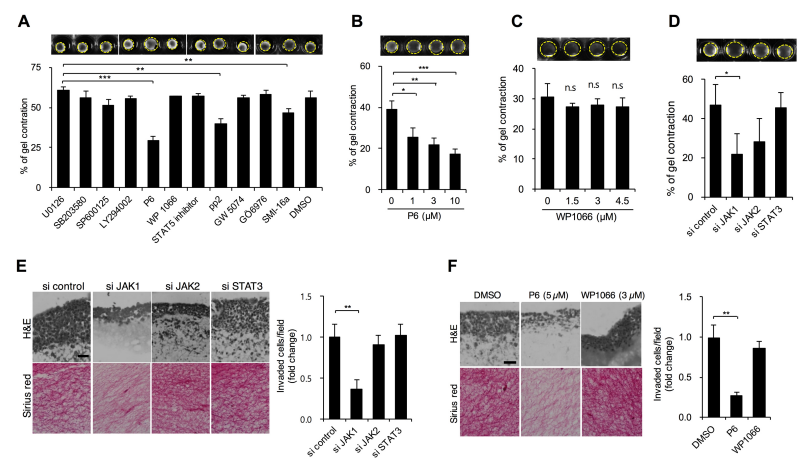

Fig. 2. JAK1 activation is required for ECM remodeling ability of tMSLCs. (A) Quantification of matrix contraction by tMSLC0903 treated with pharmacological inhibitors against oncogenic signaling components as indicated. (B-D) Quantification of matrix contraction by tMSLC0903 treated with pan-JAK inhibitor P6 (B), JAK2/STAT3 inhibitor WP1066 (C), or with siRNAs against JAK1 (D). (E) H\&E staining (upper panel) and collagen staining with Sirius red (lower panel). Quantification of GBM cell invasion in collagen-based matrix preconditioned by TMSLCs treated with siRNAs as indicated (right panel). (F) H\&E staining (upper panel) and collagen staining with Sirius red (lower panel). Quantification of X01 GBM cell invasion in collagen-based matrix preconditioned by tMSLCs treated with pan-JAK inhibitor P6 or JAK2/STAT3 inhibitor WP1066 (right panel). Data are presented as mean \pm SD from one of three independent experiments performed. ${ }^{*} \mathrm{P}<0.05$ vs. control; $* * \mathrm{P}<$ 0.01 vs. control; $* * * P<0.001$ vs. control. 
force-mediated ECM remodeling, we next sought to define the signaling factor that regulates contractile force in tMSLCs. After treatment with various chemical inhibitors against signaling pathways involved in GBM progression, we found that inhibition of JAK, SRC, or PIM1/2 suppresses the ability of tMSLCs to contract collagen gels (Fig. 2A). More significantly, treatment with the pan-JAK inhibitor P6 effectively decreased gel contraction in a dose-dependent manner, indicating that JAK $1 / 2$ in TMSLCs can be a critical regulator for ECM remodeling (Fig. 2B and Fig. S1A). Meanwhile, treatment with WP1066 inhibiting both JAK2 and STAT3 did not affect gel contraction (Fig. 2C and Fig. S1B). In parallel with these results, treatment with siRNAs (small interfering RNA) against JAK $1 / 2$ attenuated the ability of tMSLCs to contract collagen matrix, albeit to a lesser extent in the case of JAK2 siRNA (Fig. 2D). Consistently, when JAK1 was depleted, tMSLCs lost the ability to provide a proinvasive ECM microenvironment (Fig. 2E). Also, when collagen was stained with Sirius red, we observed that JAK1 depletion in TMSLCs diminishes the collagen assembly in the matrix (Fig. 2E and Fig. S1C). Treatment with pan-JAK inhibitor P6 supported that observation by confirming the role of JAK in TMSLCs on proinvasive matrix remodeling and collagen assembly (Fig. 2F). However, targeting of STAT3 with siRNA or WP1066 consistently had no effect on gel contraction, proinvasive ECM remodeling, or collagen assembly (Fig. 2D-F and Fig. S1C). Collectively, our results suggest that TMSLCs provide a proinvasive ECM microenvironment through JAK1-mediated contractile force.

JAK1 activation in TMSLCs regulates actomyosin contractility Phosphorylation of myosin light chain 2 (MLC2) is well known as a molecular signature of actomyosin contractility in CAFs. Because the above data was reminiscent of actomyosin contractility by CAFs in carcinoma (11), we next examined whether JAK1 in TMSLCs is also able to regulate phosphorylation of MLC2. Abrogating JAK1 expression with two different siRNAs decreased MLC2 phosphorylation in TMSLCs (Fig. 3A). In agreement with the data, treatment with pan-JAK inhibitor P6 also reduced MLC2 phosphorylation, indicating that TMSLCs may generate force-mediated matrix remodeling in GBM through JAK1-mediated actomyosin contractility as CAFs in carcinoma (Fig. 3B).

In view of these results, we further examined whether tMSLCs in GBM are also involved in actomyosin contractility by a similar regulatory mechanism that was seen in CAFs. Because ROCK activity is regulated by JAK1 in CAFs and participated in actomyosin contractility (10), we tested whether treatment with the ROCK inhibitor Y-27632 could diminish the effect of tMSLCs on gel contraction. Importantly, the contractile force and p-MCL2 level in TMSLCs was decreased by treatment with Y-27632 in a dose-dependent manner (Fig. 3C, D). More importantly, treatment with Y-27632 diminished the ability of tMSLCs that assemble collagen and provide a proinvasive ECM condition (Fig. 3E).

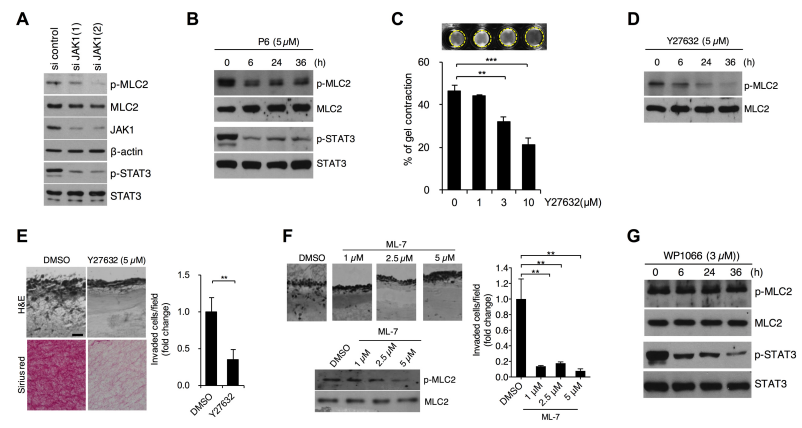

Fig. 3. JAK1 activation in tMSLCs regulates actomyosin contractility. (A, B) Western blot analysis for p-MLC2 in tMSLC0903 after treatment with siRNAs against JAK1 (A) or pan-JAK inhibitor P6 (B). $\beta$-actin was used for a loading control. (C) Quantification of matrix contraction by tMSLC0903 treated with the ROCK inhibitor Y-27632. (D) Western blot analysis for p-MLC2 in tMSLC0903 after treatment with Y-27632. (E) H\&E staining (upper panel) and collagen staining with Sirius red (lower panel). Quantification of X01 GBM cell invasion in collagen-based matrix preconditioned by tMSLCs treated with Y-27632 (right panel). (F) X01 GBM cell invasion in collagen-based matrix preconditioned by tMSLCs treated with ML-7. Representative photos are shown in upper panel. Western blot analysis for $\mathrm{p}-\mathrm{MLC2}$, a downstream effector of ML-7 in lower panel. Invasion of X01 GBM cells is quantified by counting three randomly selected microscopic fields (right panel). (G) Western blot analysis for p-MLC2 in tMSLC0903 after treatment with WP1066. Data are presented as mean \pm SD from one of three independent experiments performed. ${ }^{* * P}<0.01 \mathrm{vs}$. control; $* * * \mathrm{P}<0.001$ vs. control.

To further confirm that JAK1/ROCK-dependent contractile force occurs by MLC2-involved actomyosin contractility, we tested whether direct targeting of MLC2 phosphorylation could also attenuate the effect of tMSLCs on proinvasive ECM remodeling. To this end, we treated tMSLCs with an ML-7 myosin light chain kinase (MLCK) inhibitor during ECM remodeling in a collagen-based matrix, and then those cells were killed by puromycin and washed out before seeding with X01 GBM cells, as shown in Fig. 1C. In agreement with the above data, treatment with ML-7 markedly suppressed the effect of tMSLCs on proinvasive ECM remodeling (Fig. 3F).

However, also consistent with the above data, treatment with JAK2/STAT3 inhibitor WP1066 did not affect MLC2 phosphorylation (Fig. 3G). Because STAT3 is also a critical regulator for actomyosin contractility in CAFs, JAK1-dependent actomyosin contractility in TMSLCs is unlikely to use the same downstream effectors. Taken together, these results indicate that TMSLCs contribute to force-mediated ECM remodeling in GBM by the same actomyosin contractility that was seen in CAFs.

\section{CCL2-mediated JAK1 activation in TMSLCs regulates actomyosin contractility}

Since JAK is activated mainly by cytokines, we sought to discover cytokines that are involved in ECM remodeling. In 


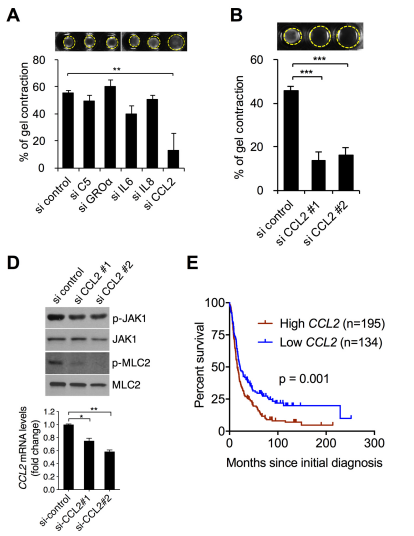

c
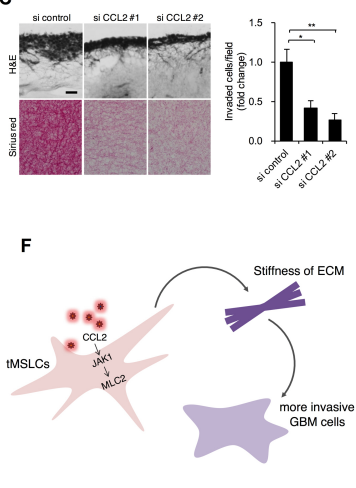

Fig. 4. CCL2-mediated JAK1 activation in tMSLCs regulates actomyosin contractility. (A) Quantification of matrix contraction by tMSLC0903 treated with siRNA as indicated. (B) Quantification of matrix contraction by tMSLC0903 treated with siRNA against CCL2. (C) H\&E staining (upper panel) and collagen staining with Sirius red (lower panel). Quantification of X01 GBM cell invasion in collagen-based matrix preconditioned by TMSLCs treated with siRNAs against CCL2 (right panel). (D) Western blot analysis for p-JAK1 and p-MLC2 in tMSLCs treated with siRNAs against CCL2 (upper). qRT-PCR was performed to validate CCL2 siRNA efficiency (lower). (E) Kaplan-Meier survival curves of patients with brain tumors in high and low levels of CCL2. (F) Schematic model illustrating force-mediated proinvasive ECM remodeling by TMSLCs in GBM microenvironment. Data are presented as mean \pm SD from one of three independent experiments performed. ${ }^{*} \mathrm{P}<0.05 \mathrm{vs}$. control; ${ }^{* *} \mathrm{P}<0.01$ vs. control; ${ }^{* * * \mathrm{P}}<0.001$ vs. control.

our previous study, we already noted the differential levels of cytokines in between tMSLCs and GBM cells (24). Because C5a, GRO alpha, IL6, IL8, and CCL2 are highly secreted from tMSLCs, we examined the effect of these cytokines on the contractile force of tMSLCs. Importantly, siRNA-mediated down-regulation of CCL2 especially reduced the contractile force of tMSLCs (Fig. 4A and Fig. S2). This result was confirmed using two different siRNA against CCL2 (Fig. 4B). Because JAK1-mediated actomyosin contractility provided proinvasive ECM, we also examined whether inhibition of CCL2 attenuates the ability of $\mathrm{MSLCs}$ that provide proinvasive ECM. As expected, treatment with CCL2 siRNA diminished the ability of tMSLCs in proinvasive ECM remodeling and collagen assembly (Fig. 4C). Accordingly, CCL2 depletion decreased p-JAK and p-MLC2 in tMSLCs (Fig. 4D). However, because CCL2 could also directly affect a phenotypic change of GBM cells into invasiveness instead of actomyosin contractility, we next examined the possibility by treatment of GBM cells with recombinant human (rh) CCL2 prior to migration assay in transwell. Treatment with rhCCL2 appears to enhance migration of GBM cells; however, it was not enough to reach statistical significance (Fig. S3).

More importantly, evaluation of data in REMBRANDT public database revealed that CCL2 levels are inversely

correlated with the survival of patient with brain tumor (Fig. $4 \mathrm{E})$, adding to the clinical importance of CCL2. Taken together, our findings reveal that TMSLCs behave as stromal cells that promote a proinvasive ECM microenvironment through CCL2/JAK1/MLC2 signal-mediated contractile force in GBM as illustrated in Fig. 4F.

\section{DISCUSSION}

ECM is commonly disorganized in cancer, consequently causing mechanical stiffness in the tumor microenvironment $(6,9)$. For a long time, the disorganization of ECM in tumors was simply regarded as a result of tumor growth. However, several strong lines of evidence have revealed that the mechanical aberrations could actively instruct malignant progression in cancer as well as providing physical track generation for migratory cancer cells $(6,11)$. In carcinoma, CAFs participate in force-mediated ECM remodeling using actomyosin contractility $(9,10)$. The contractile force generated by actomyosin contractility in CAFs has been shown to play a critical role in both collective and individual migration of cancer cells. Intriguingly, abnormal ECM dynamics resembling carcinoma is also observed in GBM; however, the stromal cells contributing to stiffness caused by ECM remodeling in GBM remain obscure.

No fibroblast has been found in the brain in contrast to the non-nervous system. Meanwhile, MSCs are present in the brain and are well known to play a role in wound tropism and to have the potential to repair damaged tissues by ECM remodeling $(20,21)$. Thus, we investigated whether tMSLCs act as the stromal cells providing the stiffness in the brain tumor microenvironment by force-mediated ECM remodeling. Notably, tMSLCs had a higher capability to contract a collagen-based gel matrix by actomyosin contractility than GBM does. Importantly, force-mediated ECM remodeling caused by TMSLCs provided a proinvasive microenvironment in GBM. The extent of gel contraction was dependent on CCL2-mediated JAK1 activation in TMSLCs. As further evidence, blocking JAK1 with either siRNA or pan-JAK inhibitor in tMSLCs effectively attenuated MLC2 phosphorylation and gel contraction, implicating that TMSLCs promote actomyosin contractility through JAK signaling. Previously, JAK1 signaling has shown to intervene in actomyosin contractility in CAFs (9, 10). In line with the previous studies, our observation underscores the pivotal role of JAK1 on actomyosin contractility.

In CAFs, depletion of STAT3 downstream of JAK1 also caused diminished levels of p-MLC2 and CAF matrixremodeling (10). In contrast, however, inhibition of STAT3 in tMSLCs had no effect on MLC2 phosphorylation, actomyosin contractility and proinvasive ECM remodeling, even though it is regulated by JAK1, indicating that force-mediated ECM remodeling is regulated through JAK1, but not STAT3 in tMSLCs. Hence, JAK1 activation is necessary for matrix remodeling in both CAFs and tMSLCs; however, they may use 
the differential downstream effectors of JAK1 for actomyosin contractility.

Aberrant cytokine signaling is a key feature of the tumor microenvironment. Since cytokine interaction with its specific receptor triggers the recruitment of JAKs to the receptors in close proximity, it is not surprising that cytokines are engaged in actomyosin contractility as addressed in previous studies (9, 10). In line with this notion, we found that CCL2 cytokine activates JAK1 in TMSLCs and participates in force-mediated matrix remodeling and thereby contributes to a proinvasive tumor microenvironment. Several strong lines of evidence have already shown the importance of CCL2 in a glioma microenvironment. Chang et al. demonstrated that CCL2 enhances the immunosuppressive tumor microenvironment by the recruitment of regulatory $\mathrm{T}$ cells and myeloid-derived suppressor cells (25). Zhang et al. reported that CCL2 promotes invasion of glioma cells via crosstalk with microglia in the tumor microenvironment (26). In line with these previous studies, our finding also adds to the importance of CCL2 in the GBM microenvironment that enhances proinvasive ECM remodeling by activating JAK1 in TMSLCs.

Recently, tMSLCs have been reported as stromal cells interacting with GBM cells, and their potential role in tumor progression has received intensive attention $(27,28)$. However, it remains controversial whether tMSLCs in the tumor microenvironment play a role in cancer promotion or suppression (29-33). These contrasting results could be caused by differences in their origin and/or the type of tumor. Thus, we previously isolated tMSLCs directly from human GBM surgical specimens and found that their presence is closely correlated with the prognosis of patients (28). In this current study, we provide another strong evidence that TMSLCs act as stromal cells promoting GBM progression by force-mediated proinvasive ECM remodeling.

In summary, our finding demonstrates that tMSLCs provide force-mediated proinvasive ECM remodeling through $\mathrm{CCL} 2 /$ JAK1/MLC2 signaling node in GBM, similar to CAFs in carcinoma. Considering the significance of CAFs in cancer progression, the mechanism underlying TMSLC-induced ECM remodeling merits further investigation in GBM.

\section{MATERIALS AND METHODS}

Detailed materials and experimental procedures are available in Supplementary Data.

\section{ACKNOWLEDGEMENTS}

We thank Akio Soeda (Department of Neurological Surgery, Gifu University, Japan) for providing patient-derived X01 GBM cells. This work was supported by the research fund of Hanyang University (HY-2017) and by the National Research Foundation (NRF) and Ministry of Science, ICT and Future Planning, Korean Government (NRF-2016R1E1A1A01942075).

\section{CONFLICTS OF INTEREST}

The authors have no conflicting interests.

\section{REFERENCES}

1. Dunn GP, Rinne ML, Wykosky J et al (2012) Emerging insights into the molecular and cellular basis of glioblastoma. Genes Dev 26, 756-784

2. Stupp R, Mason WP, van den Bent MJ et al (2005) Radiotherapy plus concomitant and adjuvant temozolomide for glioblastoma. N Engl J Med 352, 987-996

3. Dirks PB (2008) Brain tumor stem cells: bringing order to the chaos of brain cancer. J Clin Oncol 26, 2916-2924

4. Mahesparan R, Read TA, Lund-Johansen M, Skaftnesmo KO, Bjerkvig R and Engebraaten O (2003) Expression of extracellular matrix components in a highly infiltrative in vivo glioma model. Acta Neuropathol 105, 49-57

5. Bellail AC, Hunter SB, Brat DJ, Tan C and Van Meir EG (2004) Microregional extracellular matrix heterogeneity in brain modulates glioma cell invasion. Int J Biochem Cell Biol 36, 1046-1069

6. Umesh V, Rape AD, Ulrich TA and Kumar S (2014) Microenvironmental stiffness enhances glioma cell proliferation by stimulating epidermal growth factor receptor signaling. PLoS One 9, e101771

7. Grundy TJ, De Leon E, Griffin KR et al (2016) Differential response of patient-derived primary glioblastoma cells to environmental stiffness. Sci Rep 6, 23353

8. Nakada M, Nakada S, Demuth T, Tran NL, Hoelzinger DB and Berens ME (2007) Molecular targets of glioma invasion. Cell Mol Life Sci 64, 458-478

9. Albrengues J, Bourget I, Pons C et al (2014) LIF mediates proinvasive activation of stromal fibroblasts in cancer. Cell Rep 7, 1664-1678

10. Sanz-Moreno V, Gaggioli C, Yeo M et al (2011) ROCK and JAK1 signaling cooperate to control actomyosin contractility in tumor cells and stroma. Cancer Cell 20, 229-245

11. Gaggioli C, Hooper S, Hidalgo-Carcedo C et al (2007) Fibroblast-led collective invasion of carcinoma cells with differing roles for RhoGTPases in leading and following cells. Nat Cell Biol 9, 1392-1400

12. Butcher DT, Alliston T and Weaver VM (2009) A tense situation: forcing tumour progression. Nat Rev Cancer 9, 108-122

13. Unsgaard G, Rygh OM, Selbekk T et al (2006) Intraoperative 3D ultrasound in neurosurgery. Acta Neurochir (Wien) 148, 235-253; discussion 253

14. Ulrich TA, de Juan Pardo EM and Kumar S (2009) The mechanical rigidity of the extracellular matrix regulates the structure, motility, and proliferation of glioma cells. Cancer Res 69, 4167-4174

15. Calvo F, Ege N, Grande-Garcia A et al (2013) Mechanotransduction and YAP-dependent matrix remodelling is required for the generation and maintenance of cancerassociated fibroblasts. Nat Cell Biol 15, 637-646

16. Ho CM, Chang SF, Hsiao CC, Chien TY and Shih DT (2012) Isolation and characterization of stromal progenitor 
cells from ascites of patients with epithelial ovarian adenocarcinoma. J Biomed Sci 19, 23

17. Kim SM, Kang SG, Park NR et al (2011) Presence of glioma stroma mesenchymal stem cells in a murine orthotopic glioma model. Childs Nerv Syst 27, 911-922

18. Kwak J, Shin HJ, Kim SH et al (2013) Isolation of tumor spheres and mesenchymal stem-like cells from a single primitive neuroectodermal tumor specimen. Childs Nerv Syst 29, 2229-2239

19. Lim HY, Kim KM, Kim BK et al (2013) Isolation of mesenchymal stem-like cells in meningioma specimens. Int J Oncol 43, 1260-1268

20. Maxson S, Lopez EA, Yoo D, Danilkovitch-Miagkova A and Leroux MA (2012) Concise review: role of mesenchymal stem cells in wound repair. Stem Cells Transl Med 1, 142-149

21. Prockop DJ (1997) Marrow stromal cells as stem cells for nonhematopoietic tissues. Science 276, 71-74

22. Kim YG, Jeon S, Sin GY et al (2013) Existence of glioma stroma mesenchymal stemlike cells in Korean glioma specimens. Childs Nerv Syst 29, 549-563

23. Dominici M, Le Blanc K, Mueller I et al (2006) Minimal criteria for defining multipotent mesenchymal stromal cells. The International Society for Cellular Therapy position statement. Cytotherapy 8, 315-317

24. Lim EJ, Suh Y, Yoo KC et al (2017) Tumor-associated mesenchymal stem-like cells provide extracellular signaling cue for invasiveness of glioblastoma cells. Oncotarget 8, 1438-1448

25. Chang AL, Miska J, Wainwright DA et al (2016) CCL2 Produced by the Glioma Microenvironment Is Essential for the Recruitment of Regulatory T Cells and MyeloidDerived Suppressor Cells. Cancer Res 76, 5671-5682

26. Zhang J, Sarkar S, Cua R, Zhou Y, Hader W and Yong VW
(2012) A dialog between glioma and microglia that promotes tumor invasiveness through the CCL2/CCR2/ interleukin- 6 axis. Carcinogenesis 33, 312-319

27. Hossain A, Gumin J, Gao F et al (2015) Mesenchymal Stem Cells Isolated From Human Gliomas Increase Proliferation and Maintain Stemness of Glioma Stem Cells Through the IL-6/gp130/STAT3 Pathway. Stem Cells 33, 2400-2415

28. Yoon SJ, Shim JK, Chang JH et al (2016) Tumor Mesenchymal Stem-Like Cell as a Prognostic Marker in Primary Glioblastoma. Stem Cells Int 2016, 6756983

29. Xu WT, Bian ZY, Fan QM, Li G and Tang TT (2009) Human mesenchymal stem cells (hMSCs) target osteosarcoma and promote its growth and pulmonary metastasis. Cancer Lett 281, 32-41

30. Bourkoula E, Mangoni D, lus T et al (2013) Gliomaassociated stem cells: A novel class of tumor-supporting cells able to predict prognosis of human low-grade gliomas. Stem Cells 32, 1239-1253

31. Behnan J, Isakson $P$, Joel $M$ et al (2014) Recruited brain tumor-derived mesenchymal stem cells contribute to brain tumor progression. Stem Cells 32, 1110-1123

32. Otsu K, Das S, Houser SD, Quadri SK, Bhattacharya S and Bhattacharya J (2009) Concentration-dependent inhibition of angiogenesis by mesenchymal stem cells. Blood 113, 4197-4205

33. Khakoo AY, Pati S, Anderson SA et al (2006) Human mesenchymal stem cells exert potent antitumorigenic effects in a model of Kaposi's sarcoma. J Exp Med 203, 1235-1247

34. Soeda A, Park M, Lee D et al (2009) Hypoxia promotes expansion of the CD133-positive glioma stem cells through activation of HIF-1alpha. Oncogene 28, 3949-3959 